\title{
Prognostic role of preoperative carcinoembryonic antigen levels in colorectal cancer: propensity score matching
}

\author{
Cho Shin Kim, Sohyun Kim \\ Department of surgery, Yeungnam University College of Medicine, Daegu, Korea
}

\begin{abstract}
Background: This study was conducted to investigate preoperative carcinoembryonic antigen (CEA) as a prognostic factor in colorectal cancer.

Methods: Between January 2000 and July 2011, 1298 patients with primary adenocarcinoma colorectal cancer without metastasis, who underwent curative resection were retrospectively identified. The patients were divided into two groups according to serum CEA level at primary diagnosis: a high CEA (HCEA) group (serum CEA $\geq 6 \mathrm{ng} / \mathrm{mL}$ ) and a normal CEA (NCEA) group (serum CEA $<6 \mathrm{ng} / \mathrm{mL}$ ). A 1:1 propensity score matching analysis was applied to reduce bias. Finally, 364 patients were enrolled in this study. Matched variables were age, gender, preoperative chemoradiotherapy, tumor site, cell differentiation and pathologic stage.

Results: The clinicopathological characteristics of the two groups did not differ significantly difference. The systemic metastasis rate was $16.5 \%(30 / 182)$ and $25.3 \%$ (46/182) in the NCEA and HCEA groups, respectively $(p=0.039)$. There were no significant differences in local recurrence or metastatic sites between groups. The 5-year disease-free survival (DFS) rate of the HCEA group was worse than that of the NCEA group; however, there was no significant difference in overall survival between the two groups.

Conclusion: Elevated preoperative CEA was related to frequent systemic recurrence and low DFS. Therefore, elevated preoperative CEA could be considered a prognostic factor for worse clinical outcomes in patients with colorectal cancer.
\end{abstract}

Keywords: Carcinoembryonic antigen; Recurrence; Prognosis; Colorectal neoplasms

\section{INTRODUCTION}

Serum carcinoembryonic antigen (CEA) has been widely used as a tumor marker for colorectal cancer [1]. Serum CEA is usually tested at diagnosis, following treatment, and during surveillance. Serum CEA has been used as a predictive factor for early detection of recurrence after curative resection

Received: October 1, 2017, Revised: November 6, 2017 Accepted: November 6, 2017

Corresponding Author: Sohyun Kim, Department of Surgery, Yeungnam University College of Medicine, 170, Hyeonchung-ro, Nam-Gu, Daegu 42415, Korea

Tel: +82-53-620-3594, Fax: +82-53-624-1213

E-mail: kshgs@yu.ac.kr
[2,3]. Although most colorectal tumors have been found to produce CEA, elevated CEA levels are not common upon at diagnosis of patients with colorectal cancer. Overall, studies have shown that $57-66 \%$ of patients with colorectal cancer have normal preoperative CEA levels, while $34-43 \%$ of patients present with elevated preoperative CEA levels [1,2]. Elevated serum CEA is usually related to advanced tumor stage [1]. However, patients with the same tumor stage have shown inconsistency in serum CEA levels, with only some patients presenting increased CEA levels [4].

Some studies have reported a prognostic role of preoperative CEA, with elevated preoperative CEA being a poor prognostic predictor [2,5]. Specially, Ozawa et al. showed that elevated preoperative CEA was associated with worse 5-year

Copyright (C) 2017 Yeungnam University College of Medicine

This is an Open Access article distributed under the terms of the Creative Commons Attribution Non-Commercial License (http://creativecommons.org/licenses/by-nc/4.0/) which permits unrestricted non-commercial use, distribution, and reproduction in any medium, provided the original work is properly cited. 
disease-free survival (DFS) and overall survival (OS) [5]. However, in these studies, the elevated preoperative CEA group showed higher pathologic tumor $(\mathrm{T})$ and lymph node $(\mathrm{N})$ stage than the normal preoperative CEA group. Thus, elevated preoperative serum CEA was correlated with advanced stage disease [6].

Pathologic stage has been used as strong prognostic factor in colorectal cancer $[7,8]$. However, a number of authors doubted that elevated preoperative CEA indicated advanced stage colorectal cancer instead of poor prognosis. Moreover, few previous reports have suggested a prognostic role of elevated preoperative CEA in colorectal cancer, regardless of pathologic stage. Therefore, this study was conducted to investigate the prognostic role of preoperative CEA levels across all stages of colorectal cancer by applying propensity score matching.

\section{MATERIALS AND METHODS}

We conducted a retrospective review using a database of 2069 patients between January 2000 and July 2011. The inclusion criteria were as follows: (1) histologic proven adenocarcinoma; (2) curative resection of primary tumor; (3) no histological or radiological proven distant metastasis at the time of primary diagnosis; and (4) no history of hereditary, metachronous colorectal cancer or other malignancies. We eventually reviewed the medical records of 1,569 patients who followed the inclusion criteria. Patients with carcinoma in situ $(n=121)$ and those who did not have preoperative CEA $(n=150)$ were excluded. Thus, 1,298 patients were enrolled in this study. Patients were divided into two groups according to serum CEA level at the time of primary diagnosis, with those having a preoperative CEA level greater than $6 \mathrm{ng} / \mathrm{mL}$ included in the High CEA (HCEA) group and patients with a CEA level of $6 \mathrm{ng} / \mathrm{mL}$ or less included in the Normal CEA (NCEA) group.

Curative resection was defined as the absence of gross or microscopic residual tumors from the surgical bed and resection margin. Curative resection included lymph node resection at the origin of the feeding vessel. Of 1,298 patients, 682 patients underwent laparoscopic surgery and 610 patients underwent open surgery. Most patients of stage II and stage III with high risk were considered for adjuvant chemotherapy and were administered 5-fluorouracil (5-FU)/leucovorin (LV), capecitabine, or oxaliplatin. Some patients with locally advanced rectal cancer underwent radiotherapy. A total of 54 patients received preoperative radiotherapy.

All patients underwent preoperative examinations, including physical examination, laboratory tests, abdominopelvic computed tomography (CT), chest CT, and CEA blood level test. Serum CEA levels were measured using an immunoassay (Chemiluminescent Microparticle immunoassay, Abbott, USA). The normal range for serum CEA is $0-6 \mathrm{ng} / \mathrm{mL}$. Blood samples were collected 2 weeks before and 1 week after surgery. Some patients also underwent rectal or liver ultrasonography (US) and F-18 fluorodeoxyglucose positron emission tomography scan (FDG-PET); specifically, those presenting with or suspected of recurrence underwent FDG-PET. Pathologic staging was defined by the American Joint Committee on Cancer (AJCC), 7th edition. All patients underwent regular intervals follow-up after surgery that included physical examination, abdominopelvic and chest CT, and CEA blood level tests. In particular, the patients underwent follow-up CEA evaluation every 3 months for 2 years, then every 6 months for 3 years. Follow-up radiologic evaluation was performed every 6 months for 2 years, then every 12 months for 3 years. Systemic metastases were defined as metastases of distant organs such as liver and lung or peritoneum except local recurrence. Local recurrence was defined as recurrence of the previous surgical site.

Categorical variables were compared using the Chi-squared test or Fisher's exact test in both the HCEA and NCEA groups. All data were analyzed using IBM SPSS version 22.0 (IBM Co., Armonk, NY, USA). Statistical significance was defined as a $p$-value $<0.05$. Propensity score matching was used to compare significant differences in the characteristics of patients and reduce selection bias. The model was applied to obtain a one-to-one match. The following variates were matched for: age, gender, preoperative chemoradiotherapy, tumor site, cell differentiation, and pathologic stage.

\section{RESULTS}

Of the 1,298 patients, 751 (57.8 \%) were men. The mean age of the patients was 62.4 years (range, $22-88$ years) in the NCEA group and 62.6 years (range, 22-87 years) in the HCEA group. In the NCEA group, 602 (61.9\%) cases were colonic 
tumors and 371 (38.1\%) were rectal tumors. In the HCEA group, 192 (59.1\%) cases were colonic tumors and 133 (40.9\%) were rectal tumors. There were significant differences in preoperative chemoradiotherapy (PCRT), pathologic $\mathrm{T}$ stage, $\mathrm{N}$ stage, histology grade, lymphatic invasion, vascular invasion, neural invasion, and postoperative chemotherapy between the HCEA and NCEA groups (Table 1).

After propensity score matching, 364 patients (182 NCEA and 182 HCEA patients) were eligible for further study. Of the 364 patients, $228(72.6 \%)$ were men. The mean age of the patients was 63.3 years (range, 33-83 years) in both groups. There were no significant differences in any variables between groups (Table 1).

One week postoperatively, 179 patients $(98.4 \%, 179 / 182)$ in the NCEA group presented normal postoperative CEA values. In the HCEA group, 138 patients $(75.8 \%, 138 / 182)$ presented normal postoperative CEA values $(p<0.0001)$.

Overall recurrence was presented in 32 patients $(17.6 \%$, $32 / 182)$ in the NCEA group and 48 patients $(26.4 \%, 48 / 182)$ in the HCEA group $(p=0.043)$. For analysis of recurrence patterns, the site of recurrence was divided into systemic metastasis and local recurrence. Systemic metastasis presented in $30(16.5 \%)$ patients and $46(25.3 \%)$ patients in the NCEA and HCEA groups, respectively $(p=0.039)$. There were no significant differences in local recurrences and metastatic sites between groups ( $p=1.000$ and 0.829 , respectively) (Table 2).

There were significant differences in DFS between groups ( $p=0.006$, Fig. 1A). In the NCEA and HCEA groups, the 5 -year DFS was $80.4 \%$ and $67.5 \%$, respectively. However, there was no significant difference in OS between groups ( $p=0.092$, Fig. 1B). The 5 -year OS rate of the NCEA and HCEA groups was $84.4 \%$ and $78.6 \%$, respectively.

\section{DISCUSSION}

In this study, elevated preoperative CEA was associated with more frequent overall recurrence and systemic metastases regardless of tumor stage, and subsequently, with worse 5-year DFS. During the same stage, elevated preoperative CEA level was a relevant risk factor for recurrence, especially systemic metastasis. However, there was no significant difference in local recurrence and OS. Overall, the results of this study suggested that elevated preoperative CEA level is an independent prognostic factor in patients with colorectal can- cer of the same stage.

Serum CEA has been shown to be affected by various factors including stage, tumor grade, site of origin in the colon and ploidy [9]. In particular, elevated serum CEA levels have been shown to be correlated with advanced stage, with mean serum concentrations of CEA were 4.2, 6.4, 23, and $102 \mathrm{ng} / \mathrm{mL}$ in Duke's A, B, C, and D stage tumors, respectively [10]. In a previous study, the rate of an abnormal preoperative CEA ( $>5 \mathrm{ng} / \mathrm{mL}$ ) for UICC stage I, II, and III patients was $10 \%$, $47.3 \%$, and $48.6 \%$, respectively [1]. Well-differentiated colorectal cancers produce higher CEA than poorly differentiated tissues [9]. According to Bhatnagar et al., the mean CEA levels in well-differentiated, moderately-differentiated, and poorlydifferentiated colorectal adenocarcinomas were 18.0, 5.5, and $2.2 \mu \mathrm{g} / \mathrm{g}$ of protein, respectively [10]. Moreover, patients with colon tumor of the left side have been shown to have a significantly higher incidence of elevated CEA levels than those with tumors on the right side $[9,11]$. Furthermore, high levels produced by aneuploidy versus diploid patterns in colorectal tumors have been reported [9]. This study applied propensity score matching to reduce the effects of those factors on serum CEA levels and showed clinical effects of serum CEA.

Elevated preoperative CEA has been associated with overall recurrence, which has been shown in over $40 \%$ of patients with increased CEA, and 15\% of patients with low preoperative CEA $[2,12]$. In the present study, after controlling pathologic stage, patients with elevated preoperative CEA showed more frequent overall recurrence than those with normal preoperative CEA. Thus, elevated preoperative CEA could be considered a prognostic factor for recurrence in the same stage.

Many previous studies have reported that elevated preoperative CEA was related to worse DFS and OS in colorectal cancer [13,14]. Becerra et al. demonstrated that elevated preoperative CEA was associated with a $48-78 \%$ increase in death hazard [2]. Huh, et al. reported that 5-year DFS and OS were $82.4 \%$ and $81.7 \%$ in the normal CEA group and $70.6 \%$ and $69.9 \%$ in the high CEA groups, respectively [13]. In those previous studies, the elevated preoperative CEA group was found to have a larger tumor size, and more advanced $\mathrm{T}$ and $\mathrm{N}$ stage $[2,13,15]$. Moreover, $\mathrm{T}$ stage, $\mathrm{N}$ stage, cell differentiation, and high preoperative CEA were associated with worse oncologic outcomes on multivariate analysis $[2,16]$. However, another study failed to confirm the correla 
Table 1. Clinical characteristics of this study patients

\begin{tabular}{|c|c|c|c|c|c|c|}
\hline \multirow{2}{*}{ Variable } & \multicolumn{3}{|c|}{ Unmatched } & \multicolumn{3}{|c|}{ Matched } \\
\hline & NCEA $(n=973)$ & HCEA $(n=325)$ & $p$-value & $\operatorname{NCEA}(n=182)$ & HCEA $(n=182)$ & $p$-value \\
\hline Age (year) & $62.4(22-88)$ & $62.6(22-87)$ & 0.333 & $63.3(33-83)$ & $63.3(33-83)$ & 1.000 \\
\hline Gender & & & 0.701 & & & 1.000 \\
\hline Male & $560(57.6)$ & $191(58.5)$ & & $114(72.6)$ & $114(72.6)$ & \\
\hline Female & $413(42.4)$ & $134(41.2)$ & & $68(37.4)$ & $68(37.4)$ & \\
\hline ASA & & & 0.356 & & & 1.000 \\
\hline $1-2$ & $907(93.2)$ & $298(91.7)$ & & $170(93.4)$ & $168(92.3)$ & \\
\hline $3-4$ & $66(6.8)$ & $27(8.3)$ & & $12(6.6)$ & $14(7.7)$ & \\
\hline PCRT & & & $<0.001$ & & & 1.000 \\
\hline No & 907 (93.2) & $245(75.4)$ & & $155(85.2)$ & $155(85.2)$ & \\
\hline Yes & $66(6.8)$ & $80(24.6)$ & & $27(14.8)$ & $27(14.8)$ & \\
\hline Tumor site & & & 0.371 & & & 1.000 \\
\hline Col on & $602(61.9)$ & $192(59.1)$ & & $126(69.2)$ & $126(69.2)$ & \\
\hline Rectum & $371(38.1)$ & $133(40.9)$ & & $56(30.8)$ & $56(30.8)$ & \\
\hline $\mathrm{T}$ stage & & & $<0.001$ & & & 0.137 \\
\hline yp Tis & $24(2.5)$ & $7(2.2)$ & & $2(1.1)$ & $3(1.6)$ & \\
\hline $\mathrm{T} 1$ & $152(15.6)$ & $7(2.2)$ & & $8(4.4)$ & $7(3.8)$ & \\
\hline $\mathrm{T} 2$ & $162(16.6)$ & $16(4.9)$ & & $20(11.0)$ & $8(4.4)$ & \\
\hline T3 & $553(56.8)$ & 237 (72.9) & & $130(71.4)$ & $133(73.1)$ & \\
\hline $\mathrm{T} 4$ & $82(8.4)$ & $58(17.8)$ & & $22(12.1)$ & $31(17.0)$ & \\
\hline $\mathrm{N}$ stage & & & $<0.001$ & & & 0.873 \\
\hline 0 & $634(65.2)$ & $158(48.6)$ & & $89(48.9)$ & $89(48.9)$ & \\
\hline 1 & $259(26.6)$ & $116(35.7)$ & & $73(40.1)$ & $70(38.5)$ & \\
\hline 2 & $80(8.2)$ & $51(15.7)$ & & $20(11.0)$ & $23(12.6)$ & \\
\hline Histologic grade & & & 0.003 & & & 1.000 \\
\hline G1-2 & $916(94.1)$ & $290(89.2)$ & & $179(98.4)$ & $179(98.4)$ & \\
\hline G3 & $57(5.9)$ & $35(10.8)$ & & $3(1.6)$ & $3(1.6)$ & \\
\hline Lymphatic invasion & & & 0.015 & & & 0.818 \\
\hline No & $592(60.8)$ & $178(54.8)$ & & $97(53.3)$ & $93(51.1)$ & \\
\hline Yes & $360(37.0)$ & $145(44.6)$ & & $83(45.6)$ & $87(47.8)$ & \\
\hline Indeterminate & $21(2.2)$ & $2(0.6)$ & & $2(1.1)$ & $2(1.1)$ & \\
\hline Vascular invasion & & & 0.026 & & & 0.560 \\
\hline No & $875(89.9)$ & $285(87.7)$ & & $166(91.2)$ & $160(87.9)$ & \\
\hline Yes & $77(7.9)$ & $38(11.7)$ & & $15(8.2)$ & $20(11.0)$ & \\
\hline Indeterminate & $21(2.2)$ & $2(0.6)$ & & $1(0.5)$ & $2(1.1)$ & \\
\hline Neural invasion & & & $<0.001$ & & & 0.900 \\
\hline No & $744(76.5)$ & $216(66.5)$ & & $128(70.3)$ & $124(68.1)$ & \\
\hline Yes & $204(21.0)$ & $107(32.9)$ & & $52(28.6)$ & $56(30.8)$ & \\
\hline Indeterminate & $25(2.5)$ & $2(0.6)$ & & $2(1.1)$ & $2(1.1)$ & \\
\hline Harvested lymph node & $19.9 \pm 14.0$ & $22.7 \pm 13.0$ & 0.993 & $20.1 \pm 10.9$ & $23.6 \pm 13.1$ & 0.013 \\
\hline Postoperative chemotherapy & & & $<0.001$ & & & 0.457 \\
\hline No & $229(23.5)$ & $43(13.2)$ & & $29(15.9)$ & $24(13.2)$ & \\
\hline Yes & $744(76.5)$ & $282(86.8)$ & & $153(84.1)$ & $158(86.8)$ & \\
\hline Stage & & & $<0.001$ & & & 1.000 \\
\hline 0 & $21(2.2)$ & $6(1.8)$ & & $2(1.1)$ & $2(1.1)$ & \\
\hline I & $252(25.9)$ & $18(5.5)$ & & $11(6.0)$ & $11(6.0)$ & \\
\hline II & $362(37.2)$ & $136(41.9)$ & & $76(41.8)$ & $76(41.8)$ & \\
\hline III & $338(34.7)$ & $165(50.8)$ & & $93(51.1)$ & 93 (51.1) & \\
\hline
\end{tabular}

Values are presented as mean \pm standard deviation or number (\%).

NCEA, normal carcinoembryonic antigen; HCEA, high carcinoembryonic antigen; ASA, American Society of Anesthesiologists; PCRT, preoperative chemoradiotherapy. 
Table 2. Patterns of recurrence

\begin{tabular}{|c|c|c|c|}
\hline & CEA $<6(n=182)$ & CEA $\geq 6(n=182)$ & $p$-value \\
\hline Systemic metastasis & & & 0.039 \\
\hline No & $152(83.5)$ & $136(74.7)$ & \\
\hline Yes & $30(16.5)$ & $46(25.3)$ & \\
\hline Local recurrence & & & 1.000 \\
\hline No & $180(98.9)$ & $180(98.9)$ & \\
\hline Yes & $2(1.1)$ & $2(1.1)$ & \\
\hline \multicolumn{4}{|c|}{ Metastases according to organ } \\
\hline Hepatic & $11(6)$ & $12(6.6)$ & 0.829 \\
\hline Pulmonary & $7(3.8)$ & $16(8.8)$ & 0.083 \\
\hline Peritoneal & $5(2.7)$ & $5(2.7)$ & 1.000 \\
\hline Etc & $10(5.5)$ & $13(7.1)$ & 0.518 \\
\hline
\end{tabular}

Values are presented as number (\%).

CEA, carcinoembryonic antigen; Etc, et cetera.
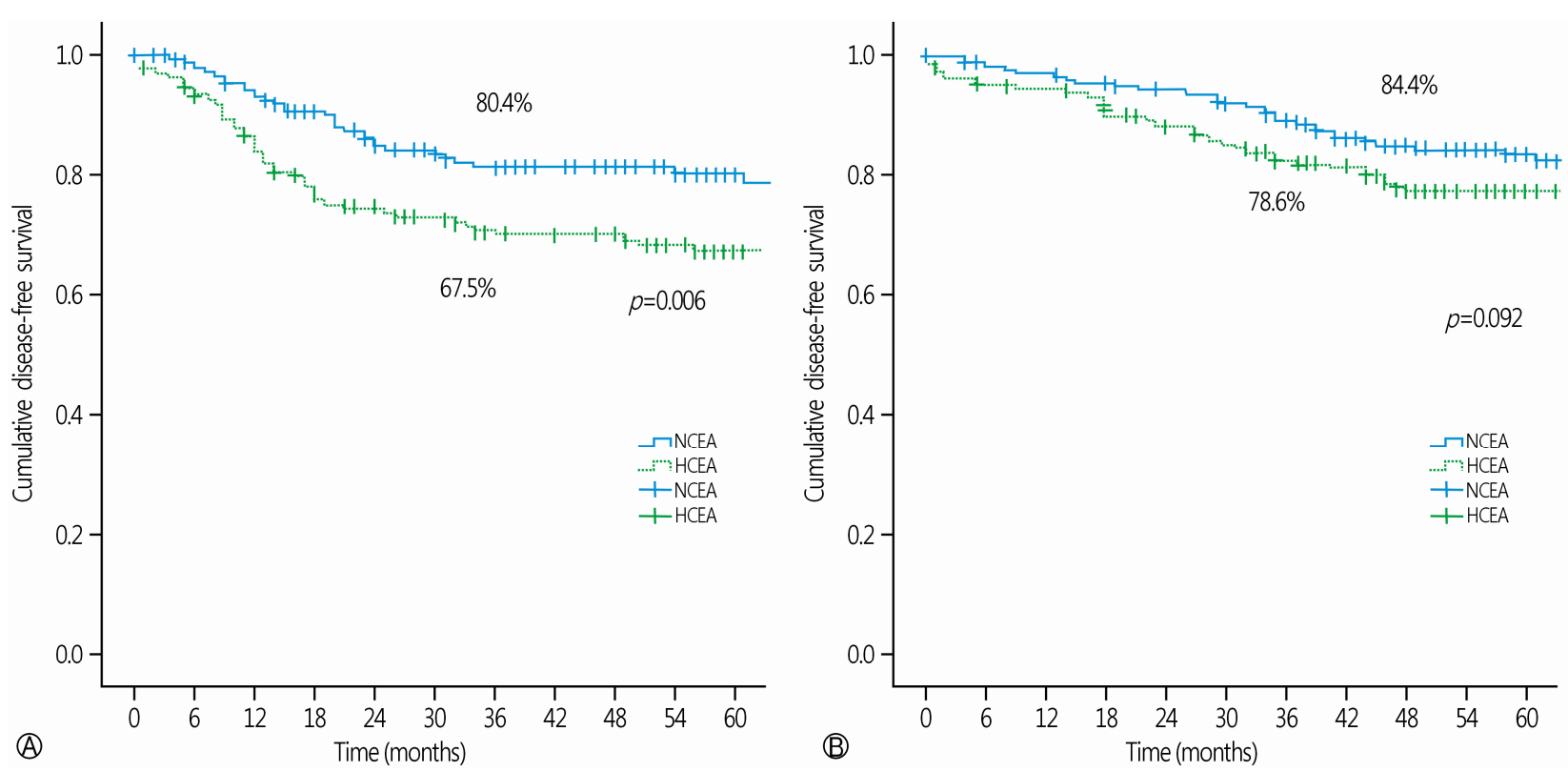

Fig. 1. (A) Five-year disease-free survival $(p=0.006)$. (B) Five-year overall survival $(p=0.092)$. NCEA, normal carcinoembryonic antigen; HCEA, high carcinoembryonic antigen.

tion between high preoperative CEA and worse clinical outcomes [15]. As previously discussed, $\mathrm{T}$ and $\mathrm{N}$ stages are important prognostic factors, while elevated preoperative serum CEA as a prognostic factor remains controversial. This study reduced the clinical effect of $\mathrm{T}$ and $\mathrm{N}$ stages, and elevated preoperative serum CEA was found to be related to worse DFS. The current study demonstrated that elevated preoperative serum CEA was not a marker for advanced tumor stage, but rather a poor prognostic factor.

In the current study, DFS was worse in the elevated preope- rative CEA group, while OS was not worse after controlling $\mathrm{T}$ and $\mathrm{N}$ stages. The authors suggest the $\mathrm{T}$ and $\mathrm{N}$ stages were more influential to OS than elevated preoperative CEA. In a previous study, a steady decrease in OS with increasing $\mathrm{T}$ stage (T1-2, 75\%; T3, 60\%; T4, 47\%; $p<0.001)$ in rectal cancer has been observed [17]. Moreover, the $\mathrm{N}$ stage has been shown to affect the 5 -year OS (N0,74\%; N1, 64\%; N2, 48\%; $p<0.001)$ [17].

It should be noted that this study has several limitations. Although the study used propensity score matching to reduce 
various biases, it is a retrospective study. The cutoff value of serum CEA was several levels according to study design $[14,16]$. Moreover, the size of this study was small. Additionally, serum CEA was likely influenced by smoking [18]; however, because of the study's retrospective nature, we could not consider the effects of smoking. We need further largescale study to evaluated role of preoperative CEA.

To conclude, our study suggests that elevated preoperative CEA level is related to high systemic recurrence and poor DFS. Moreover, elevated preoperative CEA could be a prognostic factor for worse clinical outcomes in the same stage.

\section{CONFLICT OF INTEREST}

No potential conflict of interest relevant to this article was reported.

\section{ORCID}

Sohyun Kim, http://orcid.org/0000-0002-8625-329X

\section{REFERENCES}

1. Wang JY, Lu CY, Chu KS, Ma CJ, Wu DC, Tsai HL, et al. Prognostic significance of pre- and postoperative serum carcinoembryonic antigen levels in patients with colorectal cancer. Eur Surg Res 2007;39:245-50.

2. Becerra AZ, Probst CP, Tejani MA, Aquina CT, González MG, Hensley BJ, et al. Evaluating the prognostic role of elevated preoperative carcinoembryonic antigen levels in colon cancer patients: results from the National Cancer Database. Ann Surg Oncol 2016;23:1554-61.

3. Goldstein MJ, Mitchell EP. Carcinoembryonic antigen in the staging and follow-up of patients with colorectal cancer. Cancer Invest 2005;23:338-51.

4. Moertel CG, O'Fallon JR, Go VL, O'Connell MJ, Thynne GS. The preoperative carcinoembryonic antigen test in the diagnosis, staging, and prognosis of colorectal cancer. Cancer 1986;58:603-10.

5. Ozawa H, Kotake K, Hosaka M, Hirata A, Nakagawa Y, Fujita S, et al. Incorporation of serum carcinoembryonic antigen levels into the prognostic grouping system of colon can- cer. Int J Colorectal Dis 2017;32:821-9.

6. Park IJ, Choi GS, Lim KH, Kang BM, Jun SH. Serum carcinoembryonic antigen monitoring after curative resection for colorectal cancer: clinical significance of the preoperative level. Ann Surg Oncol 2009;16:3087-93.

7. Compton CC, Fielding LP, Burgart LJ, Conley B, Cooper HS, Hamilton SR, et al. Prognostic factors in colorectal cancer. College of American Pathologists Consensus Statement 1999. Arch Pathol Lab Med 2000;124:979-94.

8. Carriquiry LA, Piñeyro A. Should carcinoembryonic antigen be used in the management of patients with colorectal cancer? Dis Colon Rectum 1999;42:921-9.

9. Duffy MJ. Carcinoembryonic antigen as a marker for colorectal cancer: is it clinically useful? Clin Chem 2001;47:624-30.

10. Bhatnagar J, Tewari HB, Bhatnagar M, Austin GE. Comparison of carcinoembryonic antigen in tissue and serum with grade and stage of colon cancer. Anticancer Res 1999;19: 2181-7.

11. Slater G, Papatestas AE, Aufses AH Jr. Preoperative carcinoembryonic antigen levels in colorectal carcinoma. Arch Surg 1979;114:52-3.

12. Park YA, Lee KY, Kim NK, Baik SH, Sohn SK, Cho CW. Prognostic effect of perioperative change of serum carcinoembryonic antigen level: a useful tool for detection of systemic recurrence in rectal cancer. Ann Surg Oncol 2006;13:645-50.

13. Huh JW, Oh BR, Kim HR, Kim YJ. Preoperative carcinoembryonic antigen level as an independent prognostic factor in potentially curative colon cancer. J Surg Oncol 2010;101: 396-400.

14. Kim CG, Ahn JB, Jung M, Beom SH, Heo SJ, Kim JH, et al. Preoperative serum carcinoembryonic antigen level as a prognostic factor for recurrence and survival after curative resection followed by adjuvant chemotherapy in stage III colon cancer. Ann Surg Oncol 2017;24:227-35.

15. Kirat HT, Ozturk E, Lavery IC, Kiran RP. The predictive value of preoperative carcinoembryonic antigen level in the prognosis of colon cancer. Am J Surg 2012;204:447-52.

16. Takagawa R, Fujii S, Ohta M, Nagano Y, Kunisaki C, Yamagishi $\mathrm{S}$, et al. Preoperative serum carcinoembryonic antigen level as a predictive factor of recurrence after curative resection of colorectal cancer. Ann Surg Oncol 2008;15:3433-9.

17. Gunderson LL, Sargent DJ, Tepper JE, Wolmark N, O’Connell $\mathrm{M}$, Begovic $\mathrm{M}$, et al. Impact of $\mathrm{T}$ and $\mathrm{N}$ stage and treatment on survival and relapse in adjuvant rectal cancer: a pooled analysis. J Clin Oncol 2004;22:1785-96.

18. Fukuda I, Yamakado M, Kiyose H. Influence of smoking on serum carcinoembryonic antigen levels in subjects who underwent multiphasic health testing and services. J Med Syst 1998; 22:89-93. 